\title{
From Autism to Autisms
}

\section{Diego Iacono*}

Neurodevelopmental Research Lab, Biomedical Research Institute of New Jersey (BRINJ) And Midatlantic Neonatology Associates (MANA), Morristown, Atlantic Health System (AHS), Morristown, New Jersey, USA

"Corresponding author: Diego Iacono, Neurodevelopmental Research Lab, Biomedical Research Institute of New Jersey (BRINJ), Cedar Knolls, Neuropathology Research, Biomedical Research Institute of New Jersey (BRINJ) And Midatlantic Neonatology Associates (MANA), Morristown, Atlantic Health System (AHS), Morristown, New Jersey, USA. Email(s): iacono@brinj.org; diego.iacono@atlantichealth.org

Citation: Iacono D (2019) From Autism to Autisms. Int J Autism \& Relat Disabil 2: 126. DOI: 10.29011/2642-3227.000026

Received Date: 24 September, 2019; Accepted Date: 01 October, 2019; Published Date: 04 October, 2019

After more than 75 years since Leo Kanner - the first child psychiatrist that used the term autism to describe the "Autistic Disturbance of Affective Contact" in his classic paper of the 1943 [1] - this unique neurodevelopmental disorder remains, roughly, a mystery in terms of both etiology and neuropathology features, and more importantly, in terms of effective treatments. Moreover, since the very first formal definition of this disorder in 1980 (DSM III) [2], other three major changes of definition have been proposed during the last three decades [3-6]. These multiple changes of the operational definition of autism generated, actually, a series of ripple effects concerning the proper (clinical criteria-based) definition of the epidemiological features, clinical measurements, behavioral and emotional assessments, clinicopathological correlations, genetic studies, neuroimaging analyses, etiopathogenetic hypotheses and animal modeling, as well as for the identification of preventive or protective factors for the autism and, ultimately, determined issues on how to measure the economic impact of this disorder across different health systems, or for establishing specific public health policies to provide guidelines to take the best possible care of individuals affected by this pervasive illness and their families.

While honored and privileged to have been proposed as the new Editor-in-Chief of International Journal of Autism \& Related Disabilities, it is my strongest wish and intention to put major efforts in making sure that the readership of this specialized journal will consider our publications as new and updated tools of knowledge and scientific acquisition in the field.

Our editorial goal is indeed to provide and share the most valuable experimental and evidence-based findings in the field of autism and autism-related conditions as well as one of the main sources of robust new findings, both for clinicians and researchers, interested in the effective advancement of the autism field and for a wider and more detailed understanding of the relationship between human brain and human behavior during development. In order to do so, we would like to emphasize that the editorial board and publisher of this journal will work hard to present to all folks interested in autism research, or people directly involved in the autism issues that is, doctors/researchers and families, a new and reliable repository of data on the autism, or autisms, jointly with any type of biological and non-biological factor confirmed to be tightly related to different forms of autism and autism-related disabilities. In fact, we strongly believe that autism is not a single disease, but rather a cluster of different diseases, and possibly different pathobiological mechanisms, with a central core of symptomatology in common across different individuals but with a wide set of different types of cognitive-behavioral declines in each single subject that is, with a vast and different gamma of behavioral and prognostic outcomes different in each affected individual. Also, we would like to emphasize, peer-reviewing process of the articles published in this journal, the medical and social urgency for the production of more and more scientific and robust data on the following aspects:

- Personalized-medicine based tools to tune the current diagnostic tools on a more accurate definition of the different types of sub-types of autism;

- Advancement of neuroimaging longitudinal studies focusing on specific autism aspects across all ages;

- Define obvious and subtle neuropathological aspects from brains of subjects across the entire autistic spectrum disorder and correlate neuropathological age-related features with clinical aspects in each individual (personalized medicine in the context of neurodevelopmental disorders);

- Detailed criteria-based description of all clinical measures that mostly disable autistic individuals with different types of 
co-morbidities also as provided by caregivers, families and patients as well;

- Provide unifying theories (biologically-based) on genetic, environmental, and behavioral aspects in primary autistic disorders vs. autism-trait characteristics in other neurodevelopmental non-primarily autistic disorders;

- Specific studies on the autisms across different sexes, races and cultures;

- Autisms and aging;

- New technologies and autisms: how high-tech tools can improve the life of autistic individuals;

- "The family corner": this journal will be open to accept letters of opinions from families, educational communities or autism-related institutions that deal, on a daily basis, with any medical, social or any other aspects of autism;

- New and effective pharmacological and non-pharmacological treatments.

Finally, the editorial board and I would like to emphasize the following strict policy points for any type of article published in International Journal of Autism \& Related Disabilities: scientific rigor and data reproducibility, ethically-reviewed procedures attention, clarity and web-openness. Also, we will pay attention to any new possible editorial initiative that could take advantage of technological innovations (including digital tools, videos, webinars, etc.) that could definitively represent an operative tool of knowledge and scientific education in the autism field and neurodevelopmental disorders in general.

\section{References}

1. Kanner L (1943) Autistic disturbances of affective contact. Nervous Child 2: 217-250.

2. APA (1980) Diagnostic and statistical manual of mental disorders DSM-III. American Psychiatric Association, Washington DC.

3. APA (1987) Diagnostic and statistical manual of mental disorders DSM-III-R. American Psychiatric Association, Washington DC.

4. APA (1994) Diagnostic and statistical manual of mental disorders DSM-IV. American Psychiatric Association, Washington DC.

5. APA (2000) Diagnostic and statistical manual of mental disorders DSM-IV-TR. American Psychiatric Association, Washington DC.

6. APA (2013) Diagnostic and statistical manual of mental disorders (5th ed.), DSM 5. American Psychiatric Association, Washington DC 